\title{
Atresia congénita bilateral de coanas en una adolescente: Reporte de un caso
}

\author{
Congenital bilateral choanal atresia in an adolescelent: A case report
}

Juan Pablo Duarte $\mathbf{S}^{1}$, César Augusto García G² , Carlos Alfonso Moreno G³ .

\begin{abstract}
RESUMEN
La atresia congénita de coanas se caracteriza por la presencia de placas que obliteran la comunicación entre la cavidad nasal y la nasofaringe desde el nacimiento. Se considera como incompatible con la vida cuando la condición es bilateral. El siguiente es el caso de una paciente femenina, de 17 años, sin síndromes asociados, con obstrucción y descarga nasal anterior bilateral, asociado a respiración oral desde el periodo neonatal. El diagnóstico de atresia bilateral de coanas fue confirmado por medio de endoscopía nasal y tomografía computarizada (TC). La imagen confirmó la presencia de placas atrésicas de composición mixta. La paciente recibió tratamiento quirúrgicamente por vía transnasal con resección de las placas y modelado de neocoana.
\end{abstract}

Palabras clave: Atresia de las coanas, obstrucción nasal, adulto, procedimientos quirúrgicos nasales.

\section{ABSTRACT}

Congenital choanal atresia is characterized by the presence of plates obliterating the communication between the nasal cavity and the nasopharynx from birth. If bilateral, this condition is incompatible with life. This following is the case of a 17-year-old female patient, without associated syndromes, with bilateral nasal obstruction and anterior discharge, associated to oral breathing, starting in her neonatal period. The diagnosis of bilateral choanal atresia was confirmed by nasal endoscopy and computed tomography (CT) scan, due to the presence of atretic plates of mixed composition. The patient was surgically treated with plate resection and modeling of a neochoana by means of a transnasal surgical approach.

Key words: Choanal atresia, Nasal obstruction, Adult, Nasal Surgical Procedures.

\footnotetext{
1 Departamento de Cirugía, Universidad Industrial de Santander. Bucaramanga, Santander, Colombia.

2 Universidad de Cartagena. Cartagena, Bolívar, Colombia.

3 Universidad Industrial de Santander. Bucaramanga, Santander, Colombia.

Los autores declaran no tener conflictos de interés.

Recibido el 4 de septiembre de 2019. Aceptado el 24 de octubre de 2019.
} 


\section{INTRODUCCIÓN}

La atresia de coanas es la anomalía congénita más común de la cavidad nasal, caracterizada por la falta de comunicación hacia la nasofaringe debido a la presencia de placas de composición variable que ocasionan obstrucción total ${ }^{1}$. Puede ser unilateral o bilateral, constituyendo en este último escenario una emergencia médica para los recién nacidos, dada su condición de respiradores nasales exclusivos; tratándose por consiguiente de una condición potencialmente letal ${ }^{2}$. El compromiso bilateral es extremadamente inusual en la población adulta, existiendo menos de 15 casos reportados en la literatura para el 2018 $38^{3-5}$. Presentamos el caso de una paciente adolescente con obstrucción nasal bilateral crónica y hallazgo de atresia bilateral de coanas, atendida en el Servicio de Otorrinolaringología del Hospital Universitario de Santander, Colombia.

\section{CASO CLÍNICO}

Paciente femenino, de 17 años de edad, quien asistió al servicio de consulta externa de otorrinolaringología por obstrucción nasal bilateral, rinorrea mucosa y respiración oral, desde el nacimiento. La madre refiere durante periodo neonatal cianosis cíclica que mejoraba con el llanto y dificultad para la alimentación. Sin antecedentes relevantes referidos. Al examen físico, muestra una hipoplasia del tercio medio facial y prognatismo con maloclusión dental clase III. A la rinoscopía anterior, desviación septal caudal izquierda con cornetes inferiores ausentes bilaterales, rinorrea mucosa bilateral; con avance de sonda Nelaton $\mathrm{N}^{0} 8$ fallido por ambas fosas nasales. La endoscopía con lente rígido reveló obliteración coanal bilateral y retención de secreciones (Figura 1A). En la tomografía computarizada (TC) de senos paranasales, se observa placas atrésicas bilaterales

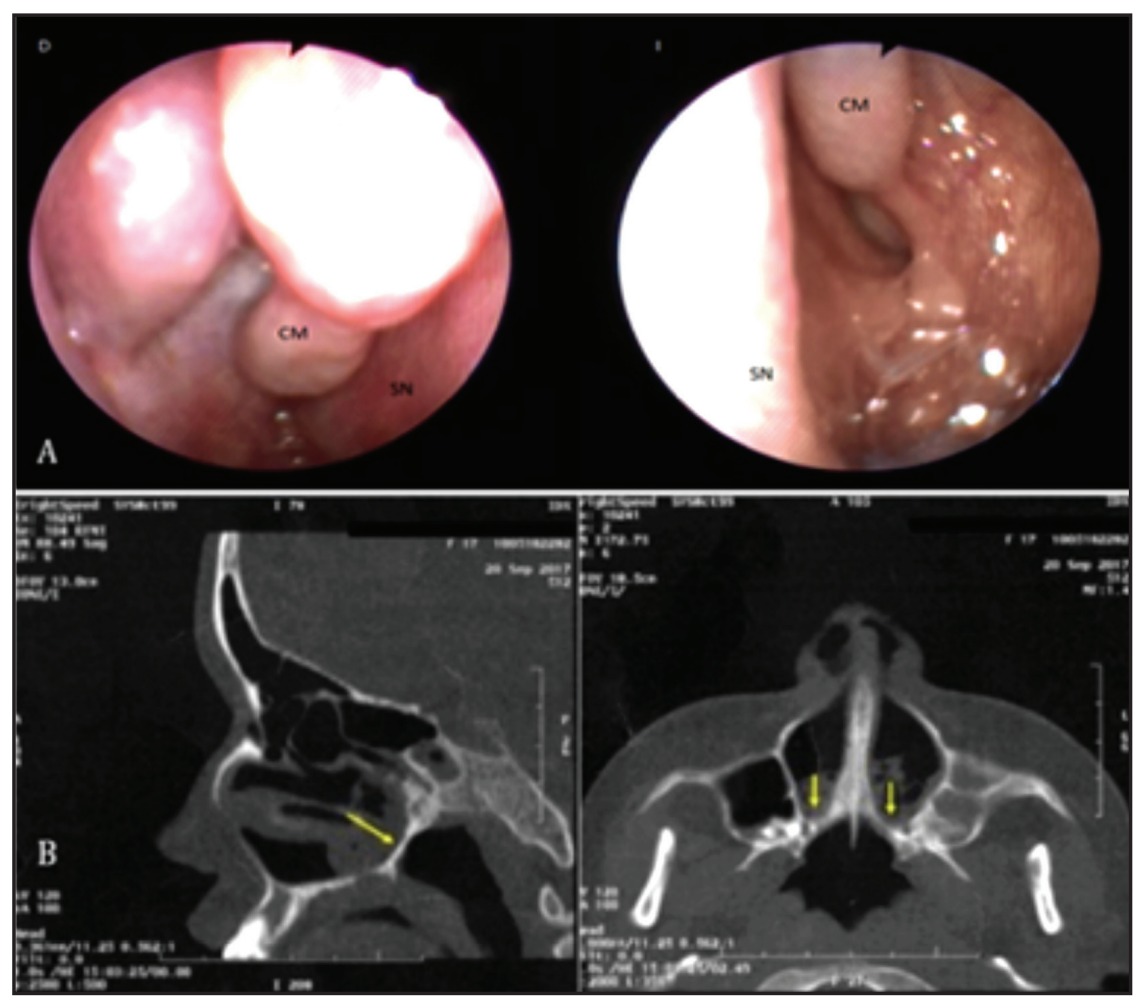

Figura 1. (A) Vista endoscópica transnasal de placa atrésica en coana derecha e izquierda; CM, cornete medio; SN, septo nasal. (B) TC de senos paranasales, corte sagital y coronal, que muestra placa atrésica mixta bilateral a nivel de coanas (flechas). 
de composición ósteo-membranosa, bloqueando el $100 \%$ de las coanas (Figura 1B), confirmando diagnóstico de atresia bilateral de coanas.

Se decide tratamiento quirúrgico por abordaje transnasal. Bajo visión endoscópica, se realizó infiltración septal posterior con lidocaína-epinefrina al $1 \%$, se diseñaron colgajos de mucosa del tercio septal posterior con pedículos invertidos superior e inferior; se realizó septectomía posterior con fresa cortante. Se resecó el componente atrésico membranoso con microdebridador y el componente atrésico óseo con fresado cortante hasta proceso pterigoideo medial bilateral, paladar duro y piso de esfenoides, obteniéndose una permeabilidad coanal completa. Se reposicionaron los colgajos mucosos con técnica cross-over, estabilizándolos con sellante de fibrina, recubriendo zonas cruentas de techo y piso en región de septectomía, para evitar osteítis y reestenosis (Figura 2).

Se aplicó mitomicina C (tópica) $0,5 \mathrm{mg} / \mathrm{ml} \mathrm{du}$ rante 3 minutos en bordes cruentos y se posicionó sonda Foley N $N^{0} 10$ en neocoana a modo de molde, insuflando el balón con 10 cc de solución salina, que fue retirada a las 48 horas. Se dio egreso con indicación de realizar irrigaciones nasales con solución salina isotónica y amoxicilina por 7 días. Se realizó control posoperatorio a los seis meses, evidenciando mejoría significativa de los síntomas y, el examen endoscópico confirmó neocoana permeable y funcional (Figura 3 ).

\section{DISCUSIÓN}

La atresia de coanas fue descrita en 1755 por J.G. Roederer ${ }^{1,6}$. Se estima que, en promedio, afecta a uno de cada 5.000 a 8.000 nacidos vivos, siendo más frecuente en el sexo femenino ${ }^{6}$. Existen diversas hipótesis desde la embriología que explican su origen, siendo las más aceptadas la persistencia de la membrana bucofaríngea, falla de la ruptura de la membrana buconasal de

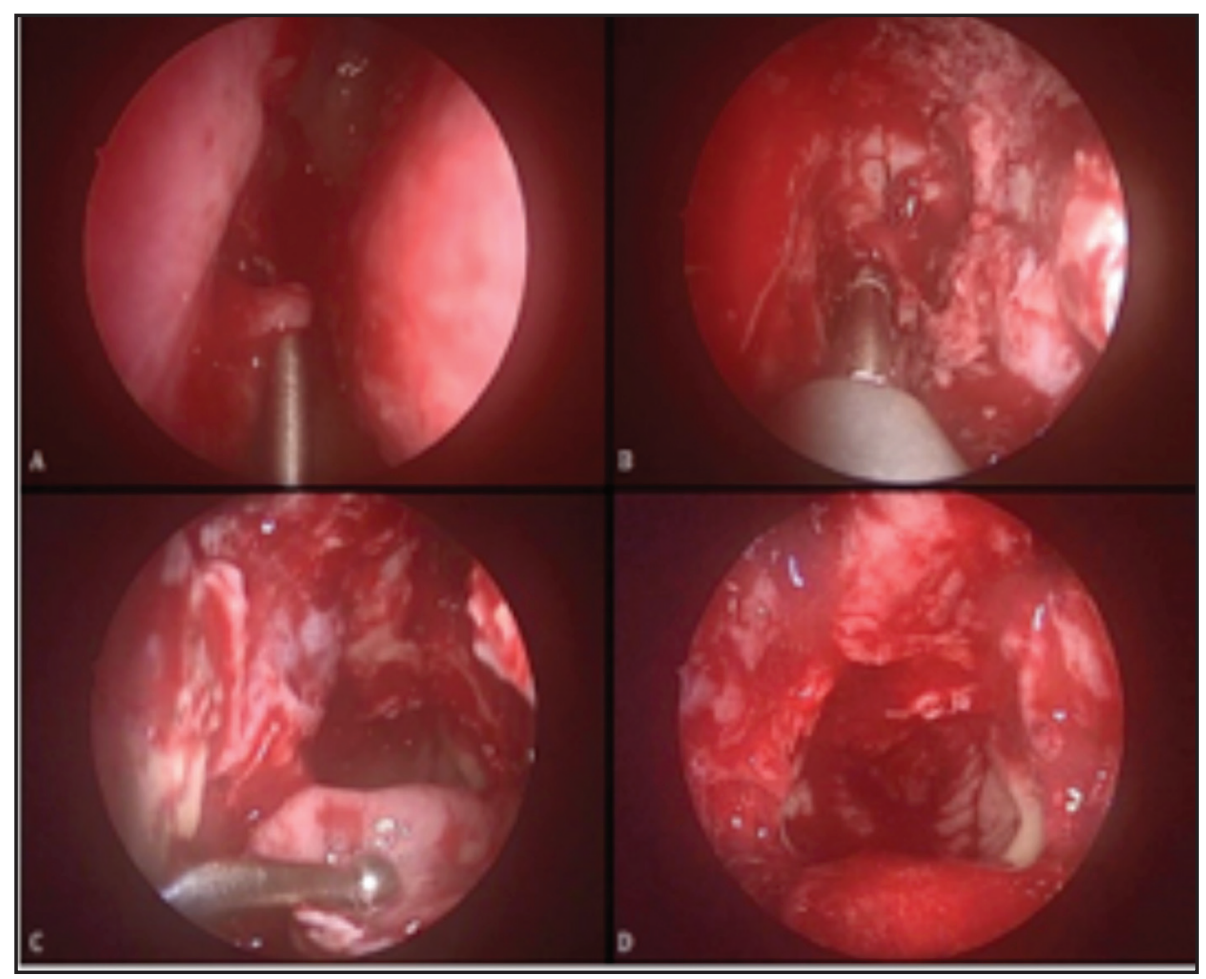

Figura 2. (A) Se diseñan colgajos de mucosa y septectomía posterior. (B) Con fresa cortante, se retira componente óseo de placa atrésica. (C) Se ubican colgajos sobre marco de neocoana. (D) Visión endoscópica de neocoana permeable. 


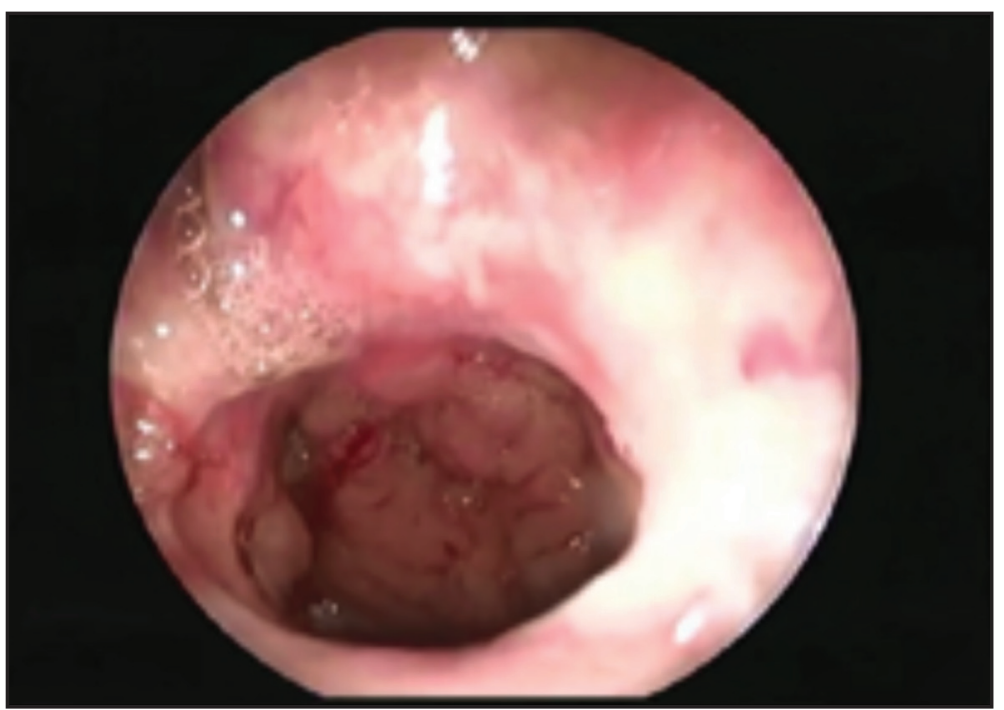

Figura 3. Vista endoscópica transnasal de neocoana permeable y epitelizada, seis meses posteriores a la cirugía.

Hochstetter, adherencias de tejido mesodérmico y migración anormal de células de la cresta neural7 Esto se traduce en una hipertrofia en la región del vómer junto con osificación del borde lateral de la coana a nivel de los platos pterigoideos. La atresia de coanas puede clasificarse en dos tipos según la composición de la placa atrésica: mixta u ósteomembranosa hasta en el 70\% de los casos, y ósea en el $30 \%$ restante. El compromiso unilateral es más frecuente $(50 \%-60 \%)$ que el bilateral ${ }^{8}$. Puede ser una entidad aislada, especialmente en los casos unilaterales; sin embargo, hasta $75 \%$ de las atresias bilaterales están asociadas a otras anomalías congénitas, como síndrome de CHARGE, TreacherCollins, Crouzon, Pfeiffer y craneosinostosis 9 .

Las manifestaciones clínicas dependen del grado de obstrucción; la atresia unilateral puede pasar desapercibida llevando a diagnósticos tardíos, incluso en edad adulta. Generalmente, consultan por obstrucción nasal y rinorrea posicional unilateral, además retención de secreciones en el fondo de cavidad nasal. En contraste, dado que los recién nacidos son considerados respiradores nasales obligados durante sus primeras 4-6 semanas de vida por la posición más cefálica y anterior de la laringe, el cuadro bilateral produce obstrucción completa de la vía aérea superior, con apneas y cia- nosis cíclica que se resuelve con el llanto, además de disfagia con alto riesgo de broncoaspiración. El riesgo de muerte por asfixia es muy elevado, por lo que es necesario estabilizar la vía aérea inmediatamente mediante el uso de chupete de McGovern, intubación orotraqueal o traqueostomía ${ }^{1,7,10}$.

Pese a tener obstrucción bilateral, la paciente no presentó los síntomas clásicos (como los descritos anteriormente) durante el periodo neonatal, ni requirió medidas avanzadas para permeabilizar la vía aérea. Esto se sustenta en el trabajo de Rodenstein y cols ${ }^{11}$ quienes demostraron que los neonatos pueden recurrir a la respiración oral, mediante la separación del paladar blando y la base de la lengua para abrir el istmo orofaríngeo cuando hay oclusión nasal (similar a los adultos que presentan apnea del sueño); sin embargo, no todos logran mantener el flujo aéreo indeterminadamente. Así mismo, no hubo antecedentes 0 alteraciones adicionales en el examen físico sugerentes de síndromes asociados. En el neonato se debe sospechar obliteración de las coanas cuando no es posible avanzar una sonda Nelaton de 6-8 Fr una distancia mayor de 3 a 3,5 cm desde el ala nasal. Actualmente, la endoscopía y la TC de senos paranasales son los métodos diagnósticos confirmatorios más utilizados y aceptados, 
resaltando que el último ofrece información sobre ubicación, composición y grosor de la placa atrésica. El uso combinado de técnicas endoscópicas y radiológicas permite la evaluación de diagnósticos diferenciales del síndrome obstructivo nasal, como la hipertrofia de cornetes, desviación septal, estenosis de apertura piriforme y lesiones asociadas a defectos de la línea media (meningoencefaloceles, gliomas, quistes dermoides) $)^{4,12}$

El tratamiento definitivo es quirúrgico. A lo largo de los años se han utilizado diferentes vías de abordaje para la corrección de la atresia de coanas (transpalatal, transantral, transeptal y transnasal), sin embargo, no existe un consenso entre los cirujanos sobre cuál es el mejor de acuerdo a su efectividad y perfil de seguridad ${ }^{13,14}$. La reparación por vía transnasal endoscópica, empleada en nuestra paciente, es la más usada en la actualidad, debido a los avances en la técnica endoscópica y los beneficios que ofrece sobre los otros métodos: visión directa del defecto, menor sangrado y riesgo de lesión a estructuras vecinas, estancia hospitalaria corta, ausencia de alteraciones cosméticas faciales y mínima afectación de los centros de crecimiento del paladar óseo y la pirámide nasal. Además, es el tratamiento quirúrgico de elección en pacientes de mayor edad ${ }^{14,15}$. La principal desventaja del abordaje endoscópico radica en la limitada manipulación de la placa atrésica cuando la cavidad nasal es estrecha, especialmente en pacientes más jóvenes, lo cual puede relacionarse con una mayor tasa de reestenosis ${ }^{16}$.

En este caso, se usó una sonda Foley como molde durante dos días para permeabilizar la neocoana y oponer los colgajos de mucosa. Esta medida es controversial, ya que hay evidencia contradictoria en la literatura con autores a favor, sustentando que reduce el riesgo de reestenosis y mejora la cicatrización de la mucosa (recomendado por 24 a 48 horas, aunque se ha reportado uso hasta por dos semanas); y otros en contra, porque pueden producir inflamación local, formación de sinequias, infección y lesión de estructuras contiguas septo nasal ${ }^{17,18}$. Se realizó topicación con mitomicina $\mathrm{C}$, por su efecto inhibidor de la proliferación y migración de fibroblastos, disminuye la formación de tejido de granulación. Esto ha sido probado en diferentes estudios que apoya su uso con el fin de disminuir la reestenosis, sin embargo, concluyen que el beneficio no es estadísticamente significativo como para usarse rutinariamente, sin mencionar su potencial efecto carcinogénico a largo plazo $0^{7,19}$. Consideramos que el uso de estos adyuvantes en la paciente, contribuyó en la obtención de un adecuado resultado del procedimiento, evidenciado en el control posoperatorio con una neocoana permeable y funcional, sin reestenosis.

\section{CONCLUSIÓN}

La atresia bilateral de coanas es considerada una entidad incompatible con la vida, con desenlace potencialmente fatal que requiere de intervenciones inmediatas para asegurar la supervivencia del neonato. Debe sospecharse ante la presencia de un recién nacido con apneas y cianosis cíclica que se resuelven con el llanto. La confirmación diagnóstica se obtiene con endoscopía nasal y TC, con el fin de corregir el defecto en las primeras semanas de vida. Sin embargo, en casos excepcionales, como nuestra paciente, no se presentan síntomas evidentes, al adaptarse a la respiración oral, y pasa desapercibido hasta edades más avanzadas. El abordaje transnasal es el más utilizado por sus beneficios en comparación a otros métodos, y se apoya en el uso de adyuvantes para disminuir el riesgo de reestenosis (como uso de stents y medicamentos inhibidores de la migración de fibroblastos) que, pese a generar controversias, han demostrado ser un aporte en la obtención de resultados favorables en esta intervención. 


\section{BIBLIOGRAFÍA}

1. San Martín MJ, Andrade DJ. Atresia de Coanas, Revisión y Una Mirada Desde la Evidencia. Rev Otorrinolaringol Cir Cabeza Cuello 2014; 74: 81-6.

2. Tatar E, Özdek A, Akcan F, Korkmaz H. Bilateral congenital choanal atresia encountered in late adulthood. J Laryngol Otol 2012; 126: 949-51.

3. Anajar S, Hassnaoui J, Rouadi S, Abada R, Roubal M, Mahtar M. A Rare Case Report of Bilateral Choanal Atresia in an Adult. Int J Surg Case Rep 2017; 37: 127-9.

4. Dib L, Marcolini M, Grad V, Kim S. Atresia Bilateral de Coanas en la Adolescencia. Revista FASO 2017; 24: 60-6.

5. Saitabu Z, Elimath M, Moshi N, Richard E, Ntunaguzi D. Bilateral Congenital Choanal Atresia in a 16year Old Girl at Muhimbili National Hospital, Tanzania. Tanzania Journal of Health Research 2018, 20: 1-6.

6. Panda NK, Simhadri S, Ghosh S. Bilateral Choanal Atresia in an Adult: is it Compatible with Life? J Laryngol Otol 2004; 118: 244-5.

7. Kwong K. Current Updates on Choanal Atresia. Frontiers in Pediatrics 2015; 3: 1-7.

8. Schweiger C, Netto C, Kuhl G, Manica D. Retrospective Study of a Series of Choanal Atresia Patients. Int Arch Otorhinolaryngol2013; 18: 2-5.

9. Verma R, Lokesh P, Panda N. Congenital Bilateral Adult Choanal Atresia Undiagnosed Until the Second Decade: How We Did It. Allergy Rhinol 2016; 7: 82-4.

10. Newman J, Harmon P, Shirley W, Hill J, Woolley A, Wiatrak B. Operative Management of Choanal Atresia: A 15-year Experience. JAMA Otolaryngol
Head Neck Surg 2013; 139: 71-5.

11. Rodenstein DO, Perlmutter N, Stanescu DC. Infants Are Not Obligatory Nasal Breathers. Am Rev Respir Dis 1985; 131: 343-7.

12. Rodríguez $H$, Cuestas $G$, Cocciaglia A, Zanetta A. Atresia de Coanas Congénita Bilateral: Diagnóstico y Tratamiento. Revista FASO 2014; 21: 54-9.

13. Cedin AC, Atallah AN, Andriolo RB, Cruz OL, PIgnatari SN. Surgery for Congenital Choanal Atresia (Review). Cochrane Database Syst Rev 2012; 2: CD008993.

14. Saraniti C, Santangelo M, Salvago P. Surgical Treatment of Choanal Atresia with Transnasal Endoscopic Approach with Stentless Single Side-hinged Flap Technique: 5-year retrospective analysis. Braz J Otorhinolaryngol 2017; 83: 1839.

15. Rodríguez H, Cuestas G, Passali D. Experiencia de 20 Años en el Tratamiento Microquirúrgico de la Atresia de Coanas. Acta Otorrinolaringol Esp 2014; 65: 85-92.

16. Kim H, Park J, Chung H, Han D, Kim D, Lee C et AL. Clinical Features and Surgical Outcomes of Congenital choanal atresia: Factors Influencing Success from 20-year Review in an Institute. Am J Otolaryngol 2012; 33: 308-12.

17. BedweLl J, Chol S. Are Stents Necessary After Choanal Atresia Repair? Laryngoscope 2012; 122: 2365-6.

18. Nazar R, Naser A, Fullá J. Atresia de Coanas en la Edad Adulta. Rev Otorrinolaringol Cir Cabeza Cuello 2008; 68: 178-84.

19. Carter J, Lawlor C, Guarisco J. The Efficacy of Mitomycin and Stenting in Choanal Atresia Repair: A 20 Year Experience. Int J Pediatr Otorhinolaryngol 2014; 78: 307-11.

Correspondencia: Juan Pablo Duarte Silva

Carrera 39 \# 48 - 06, Matisse Condominio. Bucaramanga, Colombia

E mail: jpduartesilva@hotmail.com 